\title{
A Comparative Study of Equilibrium Equity Premium under Discrete Distributions of Jump Amplitudes
}

\author{
George M. Mukupa1, Elias R. Offen², Douglas Kunda3, Edward M. Lungu4 \\ ${ }^{1}$ Department of Mathematics and Statistics, School of Science, Engineering and Technology, Mulungushi \\ University, Kabwe, Zambia \\ ${ }^{2}$ Department of Mathematics, University of Botswana, Gaborone, Botswana \\ ${ }^{3}$ School of Science, Engineering and Technology, Mulungushi University, Kabwe, Zambia \\ ${ }^{4}$ Department of Mathematics and Applied Mathematics, Botswana International University of Science and \\ Technology, Palapye, Botswana \\ Email:gmukupa@mu.ac.zm,offen@mopipi.ub.bw,dkunda@mu.edu.zm, lungue@biust.ac.bw
}

Received 10 January 2016; accepted 26 February 2016; published 29 February 2016

Copyright (C) 2016 by authors and Scientific Research Publishing Inc.

This work is licensed under the Creative Commons Attribution International License (CC BY).

http://creativecommons.org/licenses/by/4.0/

(c) (i)

Open Access

\begin{abstract}
In this paper, we compare equilibrium equity premium under discrete distributions of jump amplitudes. In particular, we consider the binomial and gamma distributions because of their applicability in finance. For the binomial, we assume that the price movement is allowed to either increase or decrease with probability $p$ or $1-p$ respectively. $n$ is the trading period thereby forming a vector $x$ of jump sizes (shifts) whose distribution is a binomial over time. For the gamma, the jumps are taken to be rare events following a Poisson distribution whose waiting times between them follows a gamma. In both distributions, the optimal consumption of the investor is affected by the deterministic time preference function $y(t)$ but it has no effect on the diffusive and rare-events premia thereby not affecting the equilibrium equity premium. Also, for $n, k=0$, the volatility effect on the equity premium is the same in both the power and square root utility functions although the equity premium is not affected by the wealth process $V(t)$. However, the wealth process affects the equity premium of the quadratic utility fuction. We observe no significant differences in equity premium for the two discrete distributions.
\end{abstract}

\section{Keywords}

Binomial Distribution, Gamma Distribution, Jump Size, Equity Risk Premium, Jump Diffusion 


\section{Introduction}

The equity risk premium or simply equity premium, the rate by which risky stocks are expected to outperform safe fixed-income investments, such as government bonds and bills, is perhaps the most important index in finance. This is the investor's compensation for taking on the relatively higher risk of the equity market. The equity risk premium is found by subtracting the estimated bond return from the estimated stock return. In our early work, we had considered the impact of utility functions in the production economy with jumps under an arbitrary jump size and derived analytical formulae for an equity premium for the power, exponential, square root and quadratic utility functions. However, we were unable to simulate graphs because of the jump size being arbitrary. In this paper, we derive numerical formulae for an equity premium and simulate graphs by imposing a Binomial distribution on the jump sizes. We then compare the results with those obtained by simulating the Gamma distribution of Jump Amplitudes. Jump diffusion has been widely explored in the area of option pricing but little work has been done to ascertain the behaviour of equity premium under jump diffusion models.

[1]-[4] studied the Pricing of Options under Jump-Diffusion Processes, and derived the appropriate characterization of asset market equilibrium when asset prices follow jump-diffusion process. They developed the general methodology for pricing options on such assets. By imposing specific restrictions on distributions and preferences, [2] formulated a tractable option pricing model that is valid even when jump risk is systematic and non-diversifiable. The dynamic hedging strategies justifying the option pricing model were described and comparisons were made throughout to the analogous problem of pricing options under stochastic volatility.

Jump Diffusion Option Valuation in Discrete Time was proposed by [5] and later developed by [6]-[16]. Multivariate jumps were superimposed on the binomial model of [17] to obtain a model with a limiting jump diffusion process. The model proposed by [5] incorporated the early exercise feature of American options as well as arbitrary jump distributions. The model yielded an efficient computational procedure that can be implemented in practice. To illustrate the model, [5] applied it to characterize the early exercise boundary of American options with certain types of jump distributions.

This paper is related to a number of papers including [11] [18]-[24] solved for the equity premium in an economy with a robust agent that has recursive utility.

\section{The Model}

This paper is based on theoretical model of [14] and also further elaboration by [25] and [26]. Consider a Jump Diffusion process;

$$
\frac{\mathrm{d} X_{t}}{X_{t^{-}}}=\mu \mathrm{d} t+\delta \mathrm{d} B_{t}+\left(\mathrm{e}^{x}-1\right) \mathrm{d} N_{t}-\lambda \mathbf{E}\left(\mathrm{e}^{x}-1\right) \mathrm{d} t .
$$

The gamma distribution arises naturally when we consider waiting times between Poisson distributed events as relevant. It can be thought of as a waiting time between Poisson distributed events.

The probability density function is the waiting time until the $k^{\text {th }}$ Poisson event with a rate of change $\lambda$. This is given by

$$
\mathbf{P}(x)=\frac{\lambda(\lambda x)^{k-1}}{(k-1) !} \mathrm{e}^{-\lambda x}
$$

Now, for $x \sim G(k, \theta)$ where $\theta=\frac{1}{\lambda}$, the gamma probability density function is

$$
\frac{x^{k-1} \mathrm{e}^{\frac{-x}{\theta}}}{\Gamma(k) \theta^{k}}
$$

where $x$ is a vector of jump amplitudes, $k$ is the number of occurrences of an event and $(e=2.71828 \cdots)$. In our case, $k$ is the number of times we observe the jumps. We realise that if $k$ is a positive integer, $\Gamma(k)=(k-1)$ ! is the gamma function. The value $\theta=\frac{1}{\lambda}$ is the mean number of jumps per time unit and $\lambda$ is the mean time between jumps. 
We still subtract the expected value from the drift so that the process becomes more volatile and hence a martingale because its future is unexpected. If we apply Itô Lemma with Jumps we have,

$$
\mathrm{d} \ln X_{t}=\left\{\left[\mu-\lambda \mathbf{E}\left(\mathrm{e}^{x}-1\right)\right]-\frac{1}{2} \delta^{2}\right\} \mathrm{d} t+\delta \mathrm{d} B_{t}+x \mathrm{~d} N_{t} .
$$

By integration we have

$$
\ln \frac{X_{T}}{X_{t}} \equiv Y_{\tau}=\left[\mu-\lambda \mathbf{E}\left(\mathrm{e}^{x}-1\right)-\frac{1}{2} \delta^{2}\right] \tau+\delta B_{t}+\sum_{i=1}^{N_{\tau}} X_{i}, \text { for } \tau=T-t
$$

where $Y_{\tau}$ is the continuously compounded investment return over the period from time $t$ to $T$ and $\tau$ is the investment period.

Suppose also that, at the risk-free rate $\rho$, the money market account $X_{0}(t)$ is such that

$$
\mathrm{d} X_{0}(t)=\rho(t) X_{0}(t) \mathrm{d} t
$$

whose total supply is assumed to be zero. Consider here that $\rho$ is risk-free because it is the rate for the money account.

We study comparatively the general equilibriums of one investor who wishes to maximize his expected reward function

$$
\max \mathbf{E}_{t} \int_{t}^{T} y(t) U\left(r_{t}\right) \mathrm{d} t
$$

subject to

$$
\frac{\mathrm{d} V_{t}}{V_{t}}=\left[\rho+\omega \phi-\omega \lambda \mathbf{E}\left(\mathrm{e}^{x}-1\right)-\frac{r_{t}}{V_{t}}\right] \mathrm{d} t+\omega \delta \mathrm{d} B_{t}+\omega\left(\mathrm{e}^{x}-1\right) \mathrm{d} N_{t}
$$

in an economy with jumps when jump amplitudes follow the binomial and gamma distributions for some time preference function $y(t)$.

\section{Results and Discussion}

Theorem 1. If $X$ is a vector of binomially distributed jump sizes, an investor's equilibrium equity premium with CRRA power utility function $U\left(r_{t}\right)=\frac{r_{t}^{\beta}}{\beta}, 0<\beta<1$, in the production economy with jump diffusion is given by

$$
\phi=-(\beta-1) \delta^{2}+\lambda(1+(\mathrm{e}-1) p)^{n}-\lambda\left(1-p+p \mathrm{e}^{\beta}\right)^{n}-\lambda+\lambda\left(1-p+p \mathrm{e}^{\beta-1}\right)^{n}
$$

where $\phi_{\delta}=-(\beta-1) \delta^{2}$ is the diffusive risk premium and $\phi_{N}=\lambda(1+(\mathrm{e}-1) p)^{n}-\lambda\left(1-p+p \mathrm{e}^{\beta}\right)^{n}-\lambda+\lambda\left(1-p+p \mathrm{e}^{\beta-1}\right)^{n}$ is the rare-event premium.

Proof. If $X$ is a random variable with a binomial distribution, then $Y=\mathrm{e}^{X}$ is a logbinomial random variable.

In particular, if $X \sim B(n, p)$ and $Y=\mathrm{e}^{X}$ then $Y^{k}=\mathrm{e}^{k X}$. Also $\mathbf{E}\left[\mathrm{e}^{k X}\right]=m_{X}(k)$ where $m_{X}(k)$ is the moment-generating function of $X$ evaluated at $k$. Hence

$$
\mathbf{E}\left[\mathrm{e}^{k X}\right]=\left(1-p+p \mathrm{e}^{k}\right)^{n}
$$

and so

$$
\mathbf{E}\left[\mathrm{e}^{X}\right]=(1-p+p \mathrm{e})^{n}=(1+(\mathrm{e}-1) p)^{n}=m_{X}(1) .
$$

Let $X=x$ be a vector of binomially distributed jump sizes then for the power utility function of [25], the rare-event premium $\phi_{N}=\lambda E\left[\left(\mathrm{e}^{x}-1\right)\left(1-\left(\mathrm{e}^{x}\right)^{\beta-1}\right)\right]$ which is $\phi_{N}=\lambda E\left[\mathrm{e}^{x}-\mathrm{e}^{x+x(\beta-1)}-1+\mathrm{e}^{x(\beta-1)}\right]$.

Now

$$
\mathbf{E}\left[\mathrm{e}^{x+\chi(\beta-1)}\right]=\mathbf{E}\left[\mathrm{e}^{x(1+\beta-1)}\right]=\mathbf{E}\left[\mathrm{e}^{\beta x}\right]=\left(1-p+p \mathrm{e}^{\beta}\right)^{n}=m_{X}(\beta) .
$$




$$
\mathbf{E}\left[\mathrm{e}^{\chi(\beta-1)}\right]=\left(1-p+p \mathrm{e}^{\beta-1}\right)^{n}=m_{X}(\beta-1) .
$$

Therefore, our rare-event premium

$$
\phi_{N}=\lambda\left[\mathbf{E}\left(\mathrm{e}^{x}\right)-\mathbf{E}\left(\mathrm{e}^{x+x(\beta-1)}\right)-1+\mathbf{E}\left(\mathrm{e}^{x(\beta-1)}\right)\right] \text { now becomes }
$$

$\phi_{N}=\lambda\left[(1+(\mathrm{e}-1) p)^{n}-\left(1-p+p \mathrm{e}^{\beta}\right)^{n}-1+\left(1-p+p \mathrm{e}^{\beta-1}\right)^{n}\right]$ which implies that our equity premium is now $\phi=-(\beta-1) \delta^{2}+\lambda(1+(\mathrm{e}-1) p)^{n}-\lambda\left(1-p+p \mathrm{e}^{\beta}\right)^{n}-\lambda+\lambda\left(1-p+p \mathrm{e}^{\beta-1}\right)^{n}$.

The optimal consumption of the investor is affected by the deterministic time preference function $y(t)$ but it has no effect on the diffusive and rare-events premia. In addition, the price of the diffusive risk $\phi_{\delta}=-(\beta-1) \delta^{2}$ is always positive for $0<\beta<1$ and $\phi_{N}=\lambda(1+(e-1) p)^{n}-\lambda\left(1-p+p \mathrm{e}^{\beta}\right)^{n}-\lambda+\lambda\left(1-p+p \mathrm{e}^{\beta-1}\right)^{n}$ is the price of the jump risk.

As can be seen in Figure 1, for $n=0$, the equity premium is almost zero when volatility is zero. This is consistent with the result for normally distributed jump sizes. Also Figure 2 shows that, as $\beta$ approach zero from the right, the equity premium increases significantly and vice-versa.

Theorem 2. For a gamma distribution of jump sizes, an investor's equilibrium equity premium with CRRA power utility function $U\left(r_{t}\right)=\frac{r_{t}^{\beta}}{\beta}, 0<\beta<1$, in the production economy with jump diffusion is given by

$$
\phi=-(\beta-1) \delta^{2}+\frac{\lambda}{\left(1-\frac{1}{\lambda}\right)^{k}}-\frac{\lambda}{\left(1-\frac{\beta}{\lambda}\right)^{k}}-\lambda+\frac{\lambda}{\left(1-\frac{(\beta-1)}{\lambda}\right)^{k}}
$$

where $\phi_{\delta}=-(\beta-1) \delta^{2}$ is the diffusive risk premium and $\phi_{N}=\frac{\lambda}{\left(1-\frac{1}{\lambda}\right)^{k}}-\frac{\lambda}{\left(1-\frac{\beta}{\lambda}\right)^{k}}-\lambda+\frac{\lambda}{\left(1-\frac{(\beta-1)}{\lambda}\right)^{k}}$ is the rare-event premium.

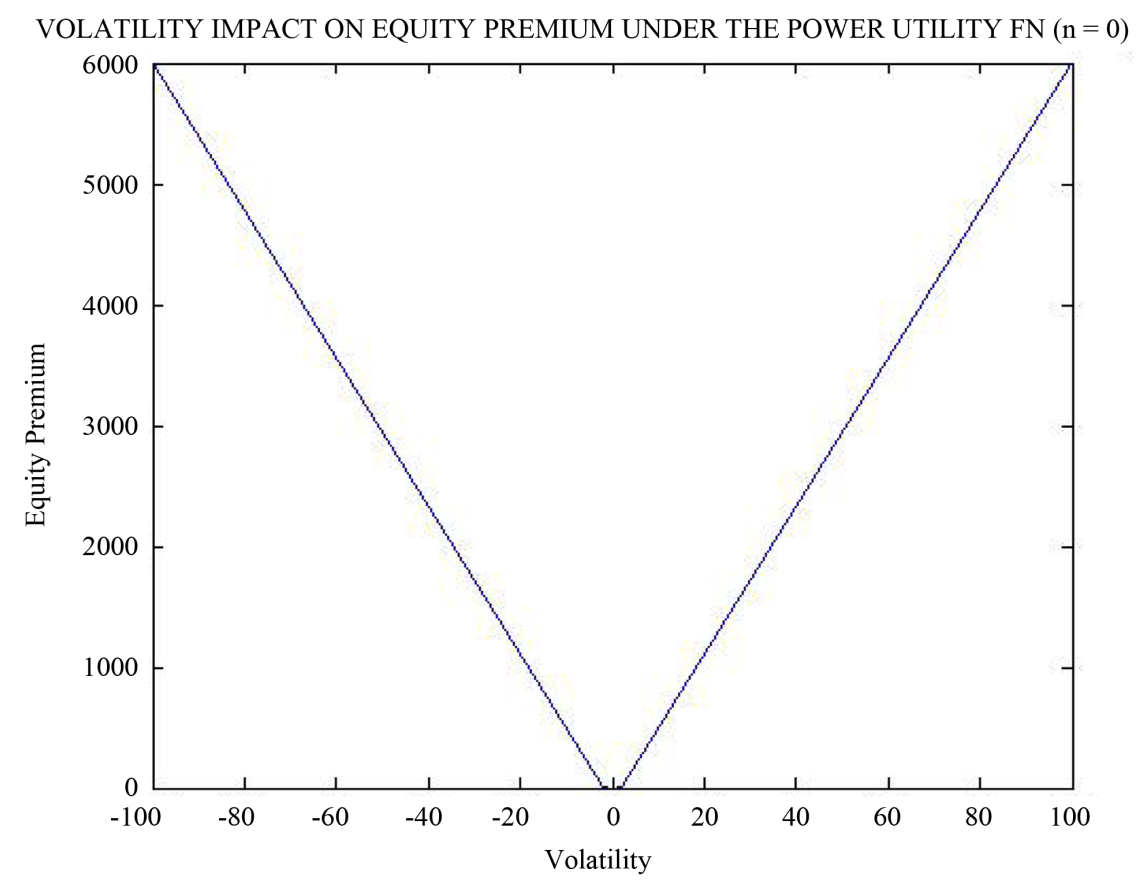

Figure 1. Power utility volatility effect under binomial distribution. 


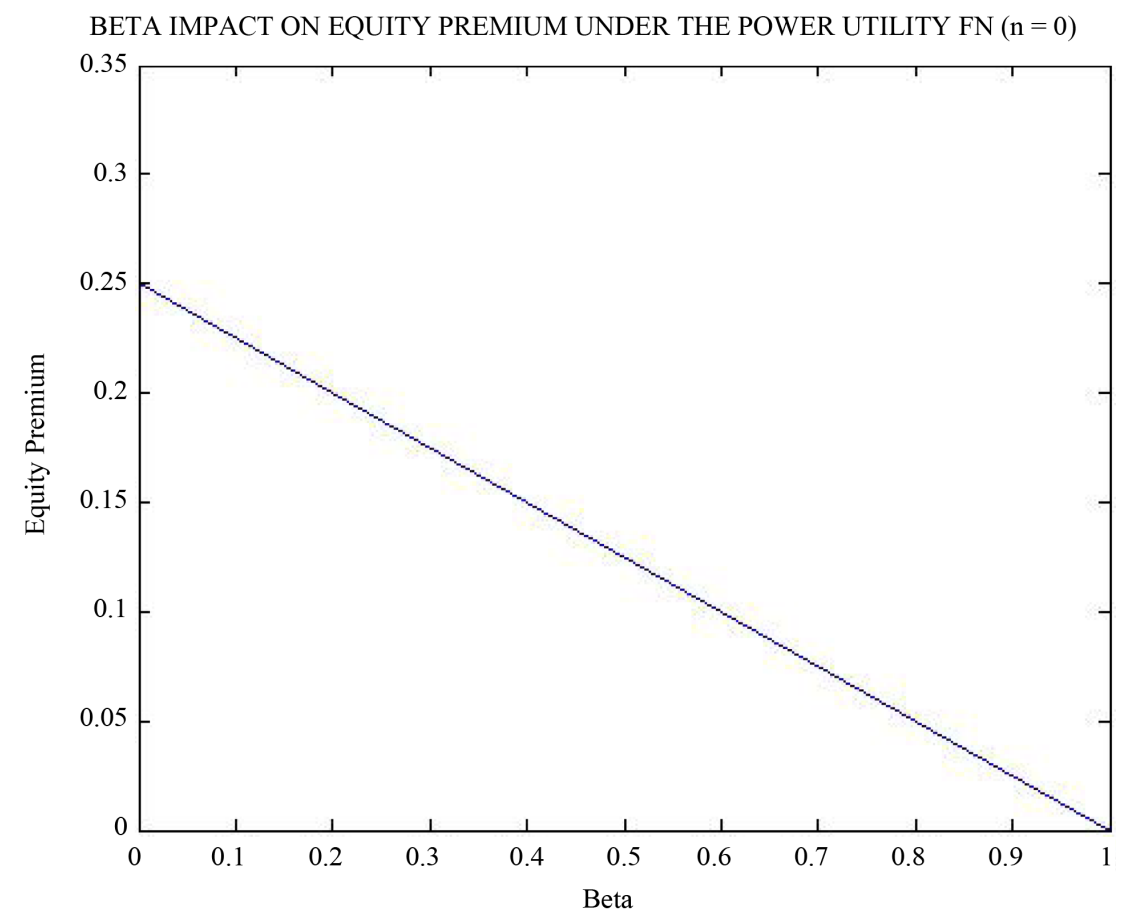

Figure 2. Power utility beta effect under binomial distribution.

Proof. If $x$ follows a gamma distribution, that is $x \sim G(\lambda, k)$ then $Y=\mathrm{e}^{x}$ is a log-gamma random variable with parameter

$$
\mathbf{E}\left[Y^{u}\right]=\mathbf{E}\left[\mathrm{e}^{u x}\right]=\frac{1}{\left(1-\frac{u}{\lambda}\right)^{k}}
$$

for some constant $u$. This is just the moment generating function of $x$ evaluated at $u$.

For the power utility function, the equilibrium equity premium $\phi$ was given by

$$
\phi=-(\beta-1) \delta^{2}+\lambda\left[\mathbf{E}\left(\mathrm{e}^{x}\right)-\mathbf{E}\left(\mathrm{e}^{x+x(\beta-1)}\right)-\mathbf{E}[1]+\mathbf{E}\left[\mathrm{e}^{x(\beta-1)}\right]\right]
$$

where our rare-event premium

$$
\phi_{N}=\lambda\left[\mathbf{E}\left(\mathrm{e}^{x}\right)-\mathbf{E}\left(\mathrm{e}^{x+\chi(\beta-1)}\right)-\mathbf{E}[1]+\mathbf{E}\left[\mathrm{e}^{\chi(\beta-1)}\right]\right]
$$

[25].

Now since $x \sim G(\lambda, k)$,

$$
\begin{gathered}
\mathbf{E}\left(\mathrm{e}^{x}\right)=\frac{1}{\left(1-\frac{1}{\lambda}\right)^{k}}=M_{x}(1) . \\
\mathbf{E}\left(\mathrm{e}^{x+\chi(\beta-1)}\right)=\mathbf{E}\left(\mathrm{e}^{\beta x}\right)=\frac{1}{\left(1-\frac{\beta}{\lambda}\right)^{k}}=M_{x}(\beta) . \\
\mathbf{E}\left(\mathrm{e}^{(\beta-1) x}\right)=\frac{1}{\left(1-\frac{(\beta-1)}{\lambda}\right)^{k}}=M_{x}(\beta-1) .
\end{gathered}
$$


Therefore our rare-event premium $\phi_{N}$ now becomes

$$
\phi_{N}=\frac{\lambda}{\left(1-\frac{1}{\lambda}\right)^{k}}-\frac{\lambda}{\left(1-\frac{\beta}{\lambda}\right)^{k}}-\lambda+\frac{\lambda}{\left(1-\frac{(\beta-1)}{\lambda}\right)^{k}}
$$

which implies that our equilibrium equity premium is

$$
\phi=-(\beta-1) \delta^{2}+\frac{\lambda}{\left(1-\frac{1}{\lambda}\right)^{k}}-\frac{\lambda}{\left(1-\frac{\beta}{\lambda}\right)^{k}}-\lambda+\frac{\lambda}{\left(1-\frac{(\beta-1)}{\lambda}\right)^{k}} .
$$

The optimal consumption of the investor is affected by the deterministic time preference function $y(t)$ but it has no effect on the diffusive and rare-events premia. In addition, the price of the diffusive risk

$\phi_{\delta}=-(\beta-1) \delta^{2}$ is always positive for $0<\beta<1$ and $\phi_{N}=\frac{\lambda}{\left(1-\frac{1}{\lambda}\right)^{k}}-\frac{\lambda}{\left(1-\frac{\beta}{\lambda}\right)^{k}}-\lambda+\frac{\lambda}{\left(1-\frac{(\beta-1)}{\lambda}\right)^{k}}$ is the price of the jump risk.

We realize in Figure 3 and Figure 4 that, for $k=n=0$, the equity premium is almost zero when the volatility is zero and the effect of beta is also the same as in the Binomial distribution respectively.

Theorem 3. In the production economy with jump diffusion under a vector of binomially distributed jump sizes, the investor's equilibrium equity premium with square root utility function $U\left(r_{t}\right)=\sqrt{r_{t}}, r_{t}>0$, is given by

$$
\phi=\frac{1}{2} \delta^{2}+\lambda(1+(\mathrm{e}-1) p)^{n}-\lambda\left(1-p+p \mathrm{e}^{\frac{1}{2}}\right)^{n}-\lambda+\lambda\left(1-p+p \mathrm{e}^{-\frac{1}{2}}\right)^{n}
$$

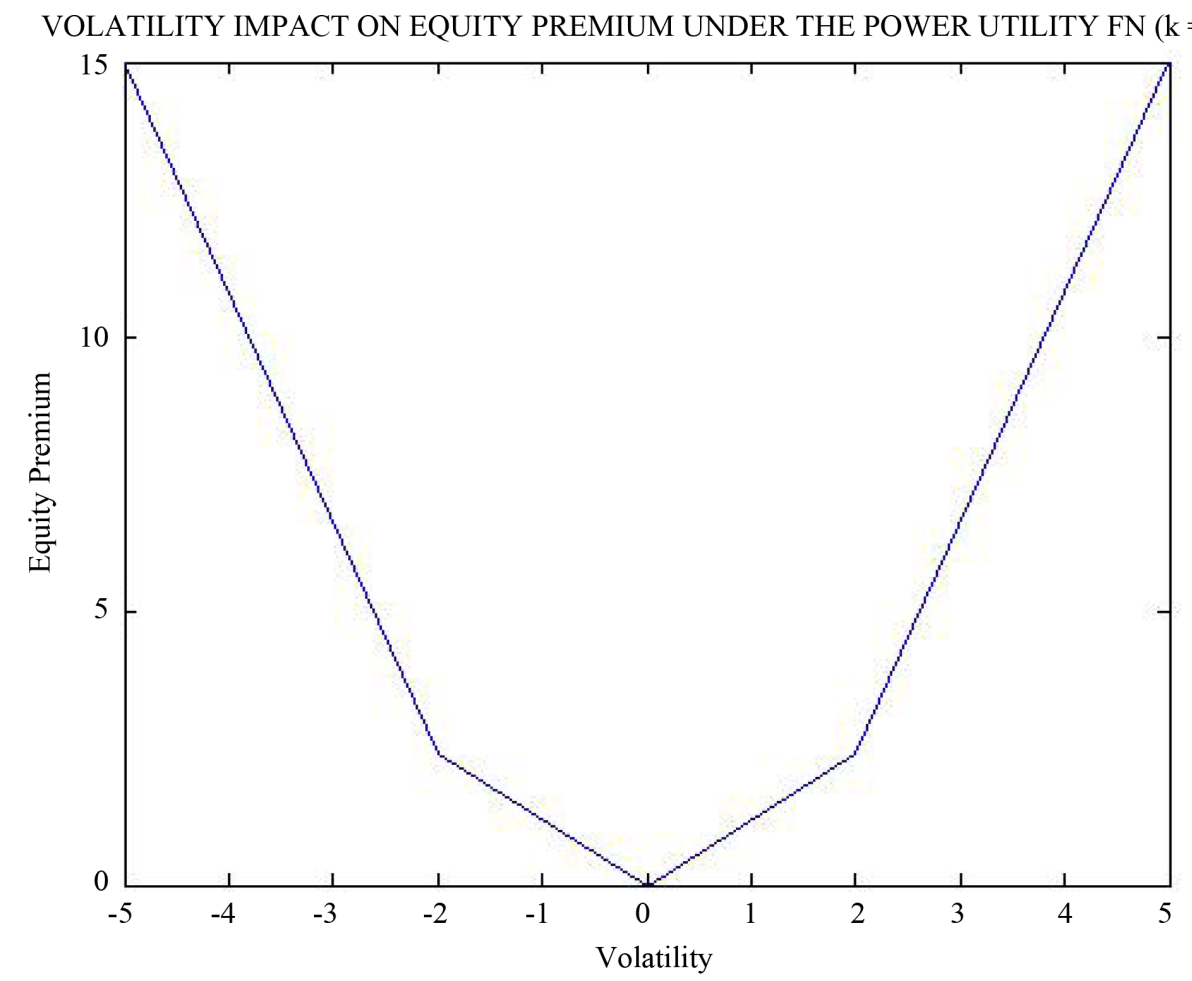

Figure 3. Power utility volatility effect under gamma distribution. 


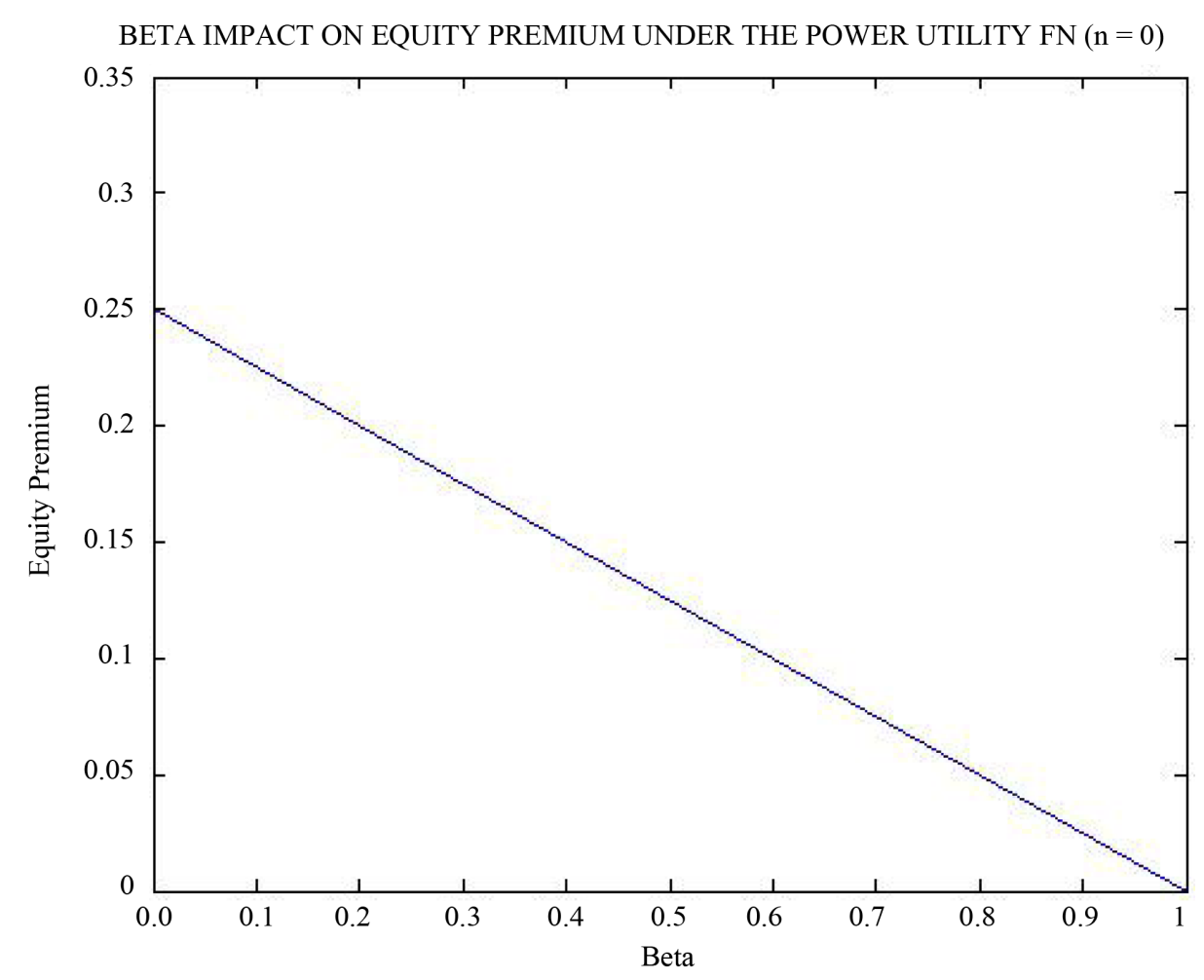

Figure 4. Power utility beta effect under gamma distribution.

where $\phi_{\delta}=\frac{1}{2} \delta^{2}$ is the diffusive risk premium and $\phi_{N}=\lambda(1+(\mathrm{e}-1) p)^{n}-\lambda\left(1-p+p \mathrm{e}^{\frac{1}{2}}\right)^{n}-\lambda+\lambda\left(1-p+p \mathrm{e}^{-\frac{1}{2}}\right)^{n}$ is the rare-event premium.

Proof. For the square root utility function, the rare-event premium is given by

$$
\begin{aligned}
\phi_{N} & =\lambda \mathbf{E}\left[\left(\mathrm{e}^{x}-1\right)\left(1-\mathrm{e}^{-\frac{1}{2} x}\right)\right]=\lambda \mathbf{E}\left[\mathrm{e}^{x}-\mathrm{e}^{\frac{1}{2} x}-1+\mathrm{e}^{-\frac{1}{2} x}\right] \\
& =\lambda\left[\mathbf{E}\left(\mathrm{e}^{x}\right)-\mathbf{E}\left(\mathrm{e}^{\frac{1}{2} x}\right)-\mathbf{E}(1)+\mathbf{E}\left(\mathrm{e}^{-\frac{1}{2} x}\right)\right] .
\end{aligned}
$$

Since $x \sim B(n, p)$, we have that

$$
\mathbf{E}\left[\mathrm{e}^{X}\right]=(1-p+p \mathrm{e})^{n}=(1+(\mathrm{e}-1) p)^{n}=m_{X}(1)
$$

and

$$
\mathbf{E}\left[\mathrm{e}^{\frac{1}{2} X}\right]=\left(1-p+p \mathrm{e}^{\frac{1}{2}}\right)^{n}=m_{X}\left(\frac{1}{2}\right)
$$

Also

$$
\mathbf{E}\left[\mathrm{e}^{-\frac{1}{2} X}\right]=\left(1-p+p \mathrm{e}^{-\frac{1}{2}}\right)^{n}=m_{X}\left(-\frac{1}{2}\right) .
$$

Thus our rare-event premium is 


$$
\lambda\left[(1+(\mathrm{e}-1) p)^{n}-\left(1-p+p \mathrm{e}^{\frac{1}{2}}\right)^{n}-1+\left(1-p+p \mathrm{e}^{-\frac{1}{2}}\right)^{n}\right]
$$

and therefore our equity premium is

$$
\phi=\frac{1}{2} \delta^{2}+\lambda(1+(\mathrm{e}-1) p)^{n}-\lambda\left(1-p+p \mathrm{e}^{\frac{1}{2}}\right)^{n}-\lambda+\lambda\left(1-p+p \mathrm{e}^{-\frac{1}{2}}\right)^{n} .
$$

The equity premium is neither affected by the wealth value nor the time preference function and the diffusive risk premium is always positive.

Just as for the power utility function and normally distributed jump size, Figure 5 suggest that, for $n=0$, the equity premium is almost zero when volatility is zero and fluctuates about a constant value when $n=55$.

Theorem 4. In the production economy with jump diffusion under a vector $x$ of jump sizes whose distribution follows a gamma, the investor's equilibrium equity premium with square root utility function $U\left(r_{t}\right)=\sqrt{r_{t}}, r_{t}>0$, is given by

$$
\phi=\frac{1}{2} \delta^{2}+\frac{\lambda}{\left(1-\frac{1}{\lambda}\right)^{k}}-\frac{\lambda}{\left(1-\frac{0.5}{\lambda}\right)^{k}}-\lambda+\frac{\lambda}{\left(1-\frac{(-0.5)}{\lambda}\right)^{k}}
$$

where $\phi_{\delta}=\frac{1}{2} \delta^{2}$ is the diffusive risk premium and $\phi_{N}=\frac{\lambda}{\left(1-\frac{1}{\lambda}\right)^{k}}-\frac{\lambda}{\left(1-\frac{0.5}{\lambda}\right)^{k}}-\lambda+\frac{\lambda}{\left(1-\frac{(-0.5)}{\lambda}\right)^{k}}$ is the rare-event premium.

Proof. For the square root utility function, the rare-event premium is given by

$$
\begin{aligned}
\phi_{N} & =\lambda \mathbf{E}\left[\left(\mathrm{e}^{x}-1\right)\left(1-\mathrm{e}^{-\frac{1}{2} x}\right)\right]=\lambda \mathbf{E}\left[\mathrm{e}^{x}-\mathrm{e}^{\frac{1}{2} x}-1+\mathrm{e}^{-\frac{1}{2} x}\right] \\
& =\lambda\left[\mathbf{E}\left(\mathrm{e}^{x}\right)-\mathbf{E}\left(\mathrm{e}^{\frac{1}{2} x}\right)-\mathbf{E}(1)+\mathbf{E}\left(\mathrm{e}^{-\frac{1}{2} x}\right)\right] .
\end{aligned}
$$

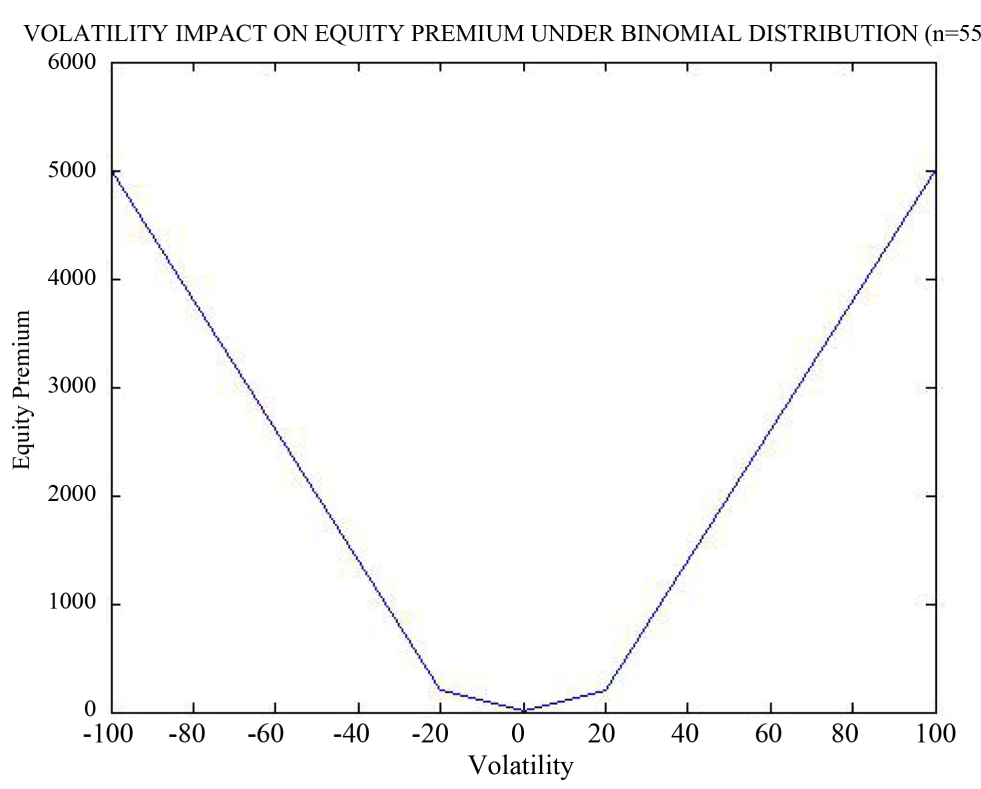

Figure 5. Square root utility volatility effect under binomial distribution. 
Now, since $x \sim G(\lambda, k)$,

$$
\begin{gathered}
\mathbf{E}\left(\mathrm{e}^{x}\right)=\frac{1}{\left(1-\frac{1}{\lambda}\right)^{k}}=M_{x}(1) \\
\mathbf{E}\left(\mathrm{e}^{\frac{1}{2} x}\right)=\frac{1}{\left(1-\frac{0.5}{\lambda}\right)^{k}}=M_{x}(0.5) \\
\mathbf{E}\left(\mathrm{e}^{-\frac{1}{2} x}\right)=\frac{1}{\left(1-\frac{(-0.5)}{\lambda}\right)^{k}}=M_{x}(-0.5)
\end{gathered}
$$

therefore

$$
\phi_{N}=\frac{\lambda}{\left(1-\frac{1}{\lambda}\right)^{k}}-\frac{\lambda}{\left(1-\frac{0.5}{\lambda}\right)^{k}}-\lambda+\frac{\lambda}{\left(1-\frac{(-0.5)}{\lambda}\right)^{k}}
$$

and thus our equilibrium equity premium is

$$
\phi=\frac{1}{2} \delta^{2}+\frac{\lambda}{\left(1-\frac{1}{\lambda}\right)^{k}}-\frac{\lambda}{\left(1-\frac{0.5}{\lambda}\right)^{k}}-\lambda+\frac{\lambda}{\left(1-\frac{(-0.5)}{\lambda}\right)^{k}} .
$$

The equity premium is neither affected by the wealth value nor the time preference function and the diffusive risk premium is always positive. For $k=0$, when volatility is zero, equity premium is zero. For $k=55$, it decreases significantly as volatility approaches zero from either side (see Figure 6). This was the case also for the power utility function.

Theorem 5. For the binomially distributed jump sizes, the investor's equilibrium equity premium with quadratic utility function $U\left(r_{t}\right)=r_{t}-a r_{t}^{2}, a>0$ in the production economy with jump diffusion is given by

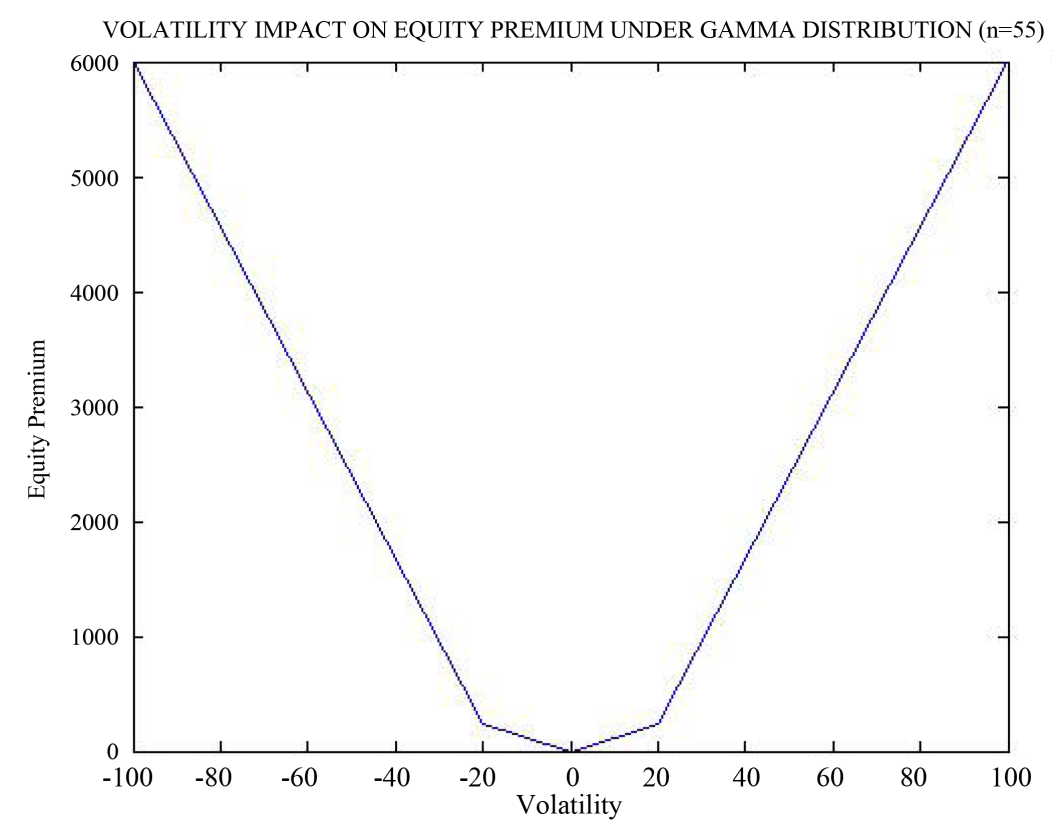

Figure 6. Square root utility volatility effect under gamma distribution. 


$$
\begin{aligned}
\phi= & \frac{2 a V_{t} \delta^{2}}{1-2 a V_{t}}+\lambda(1+(\mathrm{e}-1) p)^{n}-\frac{\lambda(1+(\mathrm{e}-1) p)^{n}}{1-2 a V_{t}}+\frac{2 a \lambda V_{t}\left(1-p+p \mathrm{e}^{2}\right)^{n}}{1-2 a V_{t}} \\
& -\lambda+\frac{\lambda}{1-2 a V_{t}}-\frac{2 a \lambda V_{t}(1+(\mathrm{e}-1) p)^{n}}{1-2 a V_{t}}
\end{aligned}
$$

where $\phi_{\delta}=\frac{2 a V_{t} \delta^{2}}{1-2 a V_{t}}$ is the diffusive risk premium and

$\phi_{N}=\lambda(1+(\mathrm{e}-1) p)^{n}-\frac{\lambda(1+(\mathrm{e}-1) p)^{n}}{1-2 a V_{t}}+\frac{2 a \lambda V_{t}\left(1-p+p \mathrm{e}^{2}\right)^{n}}{1-2 a V_{t}}-\lambda+\frac{\lambda}{1-2 a V_{t}}-\frac{2 a \lambda V_{t}(1+(\mathrm{e}-1) p)^{n}}{1-2 a V_{t}}$ is the rare-event premium.

Proof. For the HARA Quadratic utility function,

$$
\phi=\frac{2 a V_{t} \delta^{2}}{1-2 a V_{t}}+\lambda \mathbf{E}\left[\left(\mathrm{e}^{x}-1\right)\left(1-\frac{\left(1-2 a V_{t} \mathrm{e}^{x}\right)}{1-2 a V_{t}}\right)\right]
$$

where

$$
\begin{aligned}
\phi_{N} & =\lambda \mathbf{E}\left[\left(\mathrm{e}^{x}-1\right)\left(1-\frac{\left(1-2 a V_{t} \mathrm{e}^{x}\right)}{1-2 a V_{t}}\right)\right] \\
& =\lambda \mathbf{E}\left[\mathrm{e}^{x}-\frac{\mathrm{e}^{x}-2 a V_{t} \mathrm{e}^{2 x}}{1-2 a V_{t}}-1+\frac{1}{1-2 a V_{t}}-\frac{2 a V_{t} \mathrm{e}^{x}}{1-2 a V_{t}}\right] \\
& =\lambda \mathbf{E}\left[\mathrm{e}^{x}-\frac{\mathrm{e}^{x}}{1-2 a V_{t}}+\frac{2 a V_{t} \mathrm{e}^{2 x}}{1-2 a V_{t}}-1+\frac{1}{1-2 a V_{t}}-\frac{2 a V_{t} \mathrm{e}^{x}}{1-2 a V_{t}}\right] \\
& =\lambda\left[\mathbf{E}\left(\mathrm{e}^{x}\right)-\frac{\mathbf{E}\left(\mathrm{e}^{x}\right)}{1-2 a V_{t}}+\frac{2 a V_{t} \mathbf{E}\left(\mathrm{e}^{2 x}\right)}{1-2 a V_{t}}-1+\frac{1}{1-2 a V_{t}}-\frac{2 a V_{t} \mathbf{E}\left(\mathrm{e}^{x}\right)}{1-2 a V_{t}}\right] .
\end{aligned}
$$

Now since $x \sim B(n, p)$, we have that

$$
\mathbf{E}\left[\mathrm{e}^{X}\right]=(1-p+p \mathrm{e})^{n}=(1+(\mathrm{e}-1) p)^{n}=m_{X}(1)
$$

and

$$
\mathbf{E}\left[\mathrm{e}^{2 X}\right]=\left(1-p+p \mathrm{e}^{2}\right)^{n}=m_{X}(2)
$$

thus our rare-event premium is

$$
\lambda\left[(1+(\mathrm{e}-1) p)^{n}-\frac{(1+(\mathrm{e}-1) p)^{n}}{1-2 a V_{t}}+\frac{2 a V_{t}\left(1-p+p \mathrm{e}^{2}\right)^{n}}{1-2 a V_{t}}-1+\frac{1}{1-2 a V_{t}}-\frac{2 a V_{t}(1+(\mathrm{e}-1) p)^{n}}{1-2 a V_{t}}\right]
$$

which implies that our equity premium is

$$
\begin{aligned}
\phi= & \frac{2 a V_{t} \delta^{2}}{1-2 a V_{t}}+\lambda(1+(\mathrm{e}-1) p)^{n}-\frac{\lambda(1+(\mathrm{e}-1) p)^{n}}{1-2 a V_{t}}+\frac{2 a \lambda V_{t}\left(1-p+p \mathrm{e}^{2}\right)^{n}}{1-2 a V_{t}} \\
& -\lambda+\frac{\lambda}{1-2 a V_{t}}-\frac{2 a \lambda V_{t}(1+(\mathrm{e}-1) p)^{n}}{1-2 a V_{t}} .
\end{aligned}
$$

It is not affected by the time preference function $y(t)$ but is affected by $V(t)$, the total wealth of the investor at any time $t$. Figure 7 shows a constant equity premium regardless of how volatile the process 
becomes. In terms of wealth value, the equity premium is zero whenever the wealth process is zero as shown in Figure 8. This result is consistent with the normal distribution of jump sizes and maybe attributed to the fact that, for a large sample size, a discrete process maybe used to approximate a continuous process.

Theorem 6. For the gamma distribution of jump sizes, the investor's equilibrium equity premium with quadratic utility function $U\left(r_{t}\right)=r_{t}-a r_{t}^{2}, a>0$ in the production economy with jump diffusion is given by

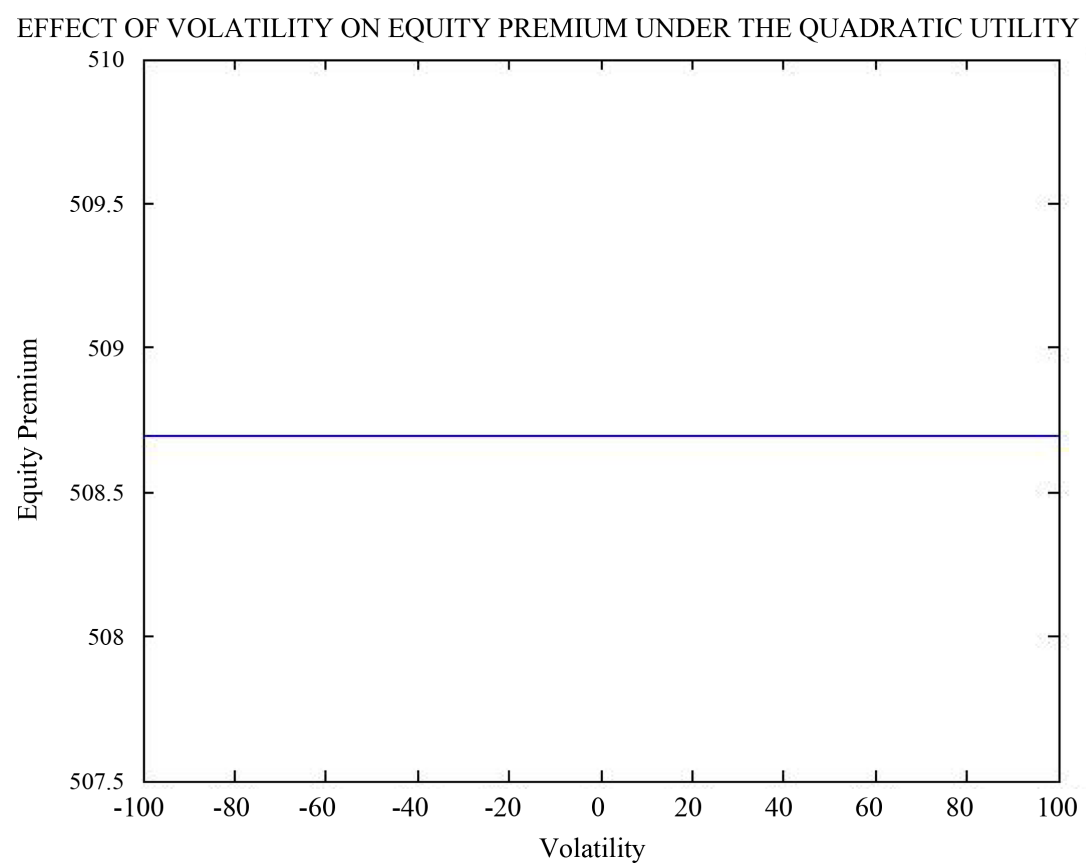

Figure 7. Quadratic utility volatility effect under binomial distribution.

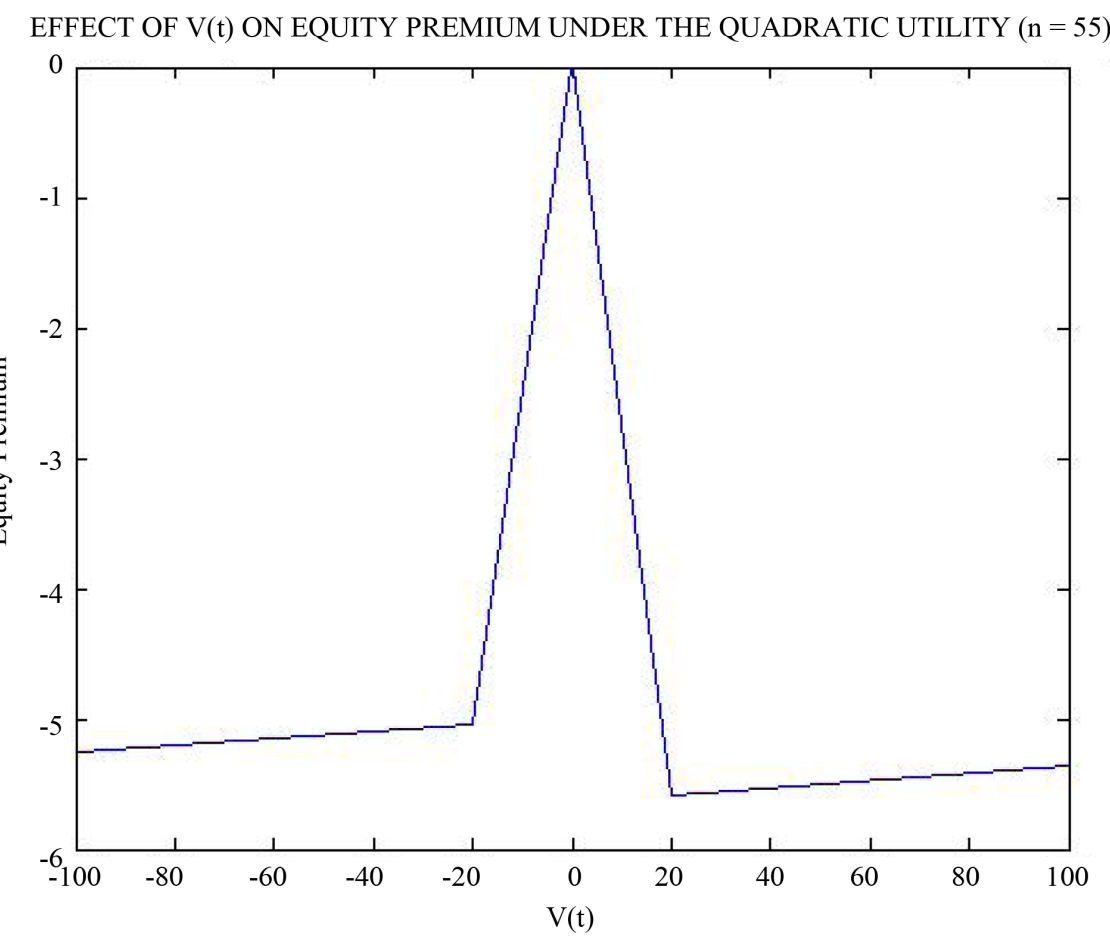

Figure 8. Quadratic utility wealth effect under binomial distribution. 


$$
\begin{aligned}
\phi= & \frac{2 a V_{t} \delta^{2}}{1-2 a V_{t}}+\frac{\lambda}{\left(1-\frac{1}{\lambda}\right)^{k}}-\frac{\lambda}{\left(1-2 a V_{t}\right)\left(1-\frac{1}{\lambda}\right)^{k}}-\frac{2 a \lambda V_{t}}{\left(1-2 a V_{t}\right)\left(1-\frac{2}{\lambda}\right)^{k}} \\
& -\lambda+\frac{\lambda}{1-2 a V_{t}}-\frac{2 a \lambda V_{t}}{\left(1-2 a V_{t}\right)\left(1-\frac{1}{\lambda}\right)^{k}}
\end{aligned}
$$

where $\phi_{\delta}=\frac{2 a V_{t} \delta^{2}}{1-2 a V_{t}}$ is the diffusive risk premium and

$\phi_{N}=\frac{\lambda}{\left(1-\frac{1}{\lambda}\right)^{k}}-\frac{\lambda}{\left(1-2 a V_{t}\right)\left(1-\frac{1}{\lambda}\right)^{k}}-\frac{2 a \lambda V_{t}}{\left(1-2 a V_{t}\right)\left(1-\frac{2}{\lambda}\right)^{k}}-\lambda+\frac{\lambda}{1-2 a V_{t}}-\frac{2 a \lambda V_{t}}{\left(1-2 a V_{t}\right)\left(1-\frac{1}{\lambda}\right)^{k}}$ is the rare-event premium.

Proof. For the HARA Quadratic utility function,

$$
\phi=\frac{2 a V_{t} \delta^{2}}{1-2 a V_{t}}+\lambda \mathbf{E}\left[\left(\mathrm{e}^{x}-1\right)\left(1-\frac{\left(1-2 a V_{t} \mathrm{e}^{x}\right)}{1-2 a V_{t}}\right)\right]
$$

where

$$
\begin{aligned}
\phi_{N} & =\lambda \mathbf{E}\left[\left(\mathrm{e}^{x}-1\right)\left(1-\frac{\left(1-2 a V_{t} \mathrm{e}^{x}\right)}{1-2 a V_{t}}\right)\right] \\
& =\lambda \mathbf{E}\left[\mathrm{e}^{x}-\frac{\mathrm{e}^{x}-2 a V_{t} \mathrm{e}^{2 x}}{1-2 a V_{t}}-1+\frac{1}{1-2 a V_{t}}-\frac{2 a V_{t} \mathrm{e}^{x}}{1-2 a V_{t}}\right] \\
& =\lambda \mathbf{E}\left[\mathrm{e}^{x}-\frac{\mathrm{e}^{x}}{1-2 a V_{t}}-\frac{2 a V_{t} \mathrm{e}^{2 x}}{1-2 a V_{t}}-1+\frac{1}{1-2 a V_{t}}-\frac{2 a V_{t} \mathrm{e}^{x}}{1-2 a V_{t}}\right] \\
& =\lambda\left[\mathbf{E}\left(\mathrm{e}^{x}\right)-\frac{\mathbf{E}\left(\mathrm{e}^{x}\right)}{1-2 a V_{t}}-\frac{2 a V_{t} \mathbf{E}\left(\mathrm{e}^{2 x}\right)}{1-2 a V_{t}}-1+\frac{1}{1-2 a V_{t}}-\frac{2 a V_{t} \mathbf{E}\left(\mathrm{e}^{x}\right)}{1-2 a V_{t}}\right] .
\end{aligned}
$$

Now since $x \sim G(\lambda, k)$, we have that

$$
\begin{aligned}
& \mathbf{E}\left(\mathrm{e}^{x}\right)=\frac{1}{\left(1-\frac{1}{\lambda}\right)^{k}}=M_{x}(1) \\
& \mathbf{E}\left(\mathrm{e}^{2 x}\right)=\frac{1}{\left(1-\frac{2}{\lambda}\right)^{k}}=M_{x}(2)
\end{aligned}
$$

thus

$$
\phi_{N}=\lambda\left[\frac{1}{\left(1-\frac{1}{\lambda}\right)^{k}}-\frac{\frac{1}{\left(1-\frac{1}{\lambda}\right)^{k}}}{1-2 a V_{t}}-\frac{2 a V_{t} \frac{1}{\left(1-\frac{2}{\lambda}\right)^{k}}}{1-2 a V_{t}}-1+\frac{1}{1-2 a V_{t}}-\frac{2 a V_{t} \frac{1}{\left(1-\frac{1}{\lambda}\right)^{k}}}{1-2 a V_{t}}\right]
$$


which is just

$$
\frac{\lambda}{\left(1-\frac{1}{\lambda}\right)^{k}}-\frac{\lambda}{\left(1-2 a V_{t}\right)\left(1-\frac{1}{\lambda}\right)^{k}}-\frac{2 a \lambda V_{t}}{\left(1-2 a V_{t}\right)\left(1-\frac{2}{\lambda}\right)^{k}}-\lambda+\frac{\lambda}{1-2 a V_{t}}-\frac{2 a \lambda V_{t}}{\left(1-2 a V_{t}\right)\left(1-\frac{1}{\lambda}\right)^{k}}
$$

So that our equilibrium equity premium is now

$$
\begin{aligned}
\phi= & \frac{2 a V_{t} \delta^{2}}{1-2 a V_{t}}+\frac{\lambda}{\left(1-\frac{1}{\lambda}\right)^{k}}-\frac{\lambda}{\left(1-2 a V_{t}\right)\left(1-\frac{1}{\lambda}\right)^{k}}-\frac{2 a \lambda V_{t}}{\left(1-2 a V_{t}\right)\left(1-\frac{2}{\lambda}\right)^{k}} \\
& -\lambda+\frac{\lambda}{1-2 a V_{t}}-\frac{2 a \lambda V_{t}}{\left(1-2 a V_{t}\right)\left(1-\frac{1}{\lambda}\right)^{k}} .
\end{aligned}
$$

It is not affected by the time preference function $y(t)$ but is affected by $V(t)$, the total wealth of the investor at any time $t$. As evident in Figure 9, although for $k=0$ the equity premium is negative, it rises significantly as the wealth value process moves from negative to zero and becomes zero when the wealth process is zero. The equity premium decreases significantly when the investor's wealth is in the range 0 to 20 and begins to rise again. For $k=55$, the wealth process $V_{t}$ affects the equity premium in the same way.

\section{Conclusions}

In conclusion, the optimal consumption of the investor is affected by the deterministic time preference function $y(t)$ but it has no effect on the diffusive and rare-events premia. For $k=0$, the equity premium is almost zero when the volatility is zero. However, it is non zero for $k=55$ even if it is symmetrical about zero premium. In fact, it decreases significantly as volatility approaches zero from either side. The equity premium for the quadratic utility function is affected by $V_{t}$ the total wealth of an investor at time $t$. When $V_{t}=0$, the equity premium is zero. However, for $k=55$, it is constant regardless of how volatile the process becomes.

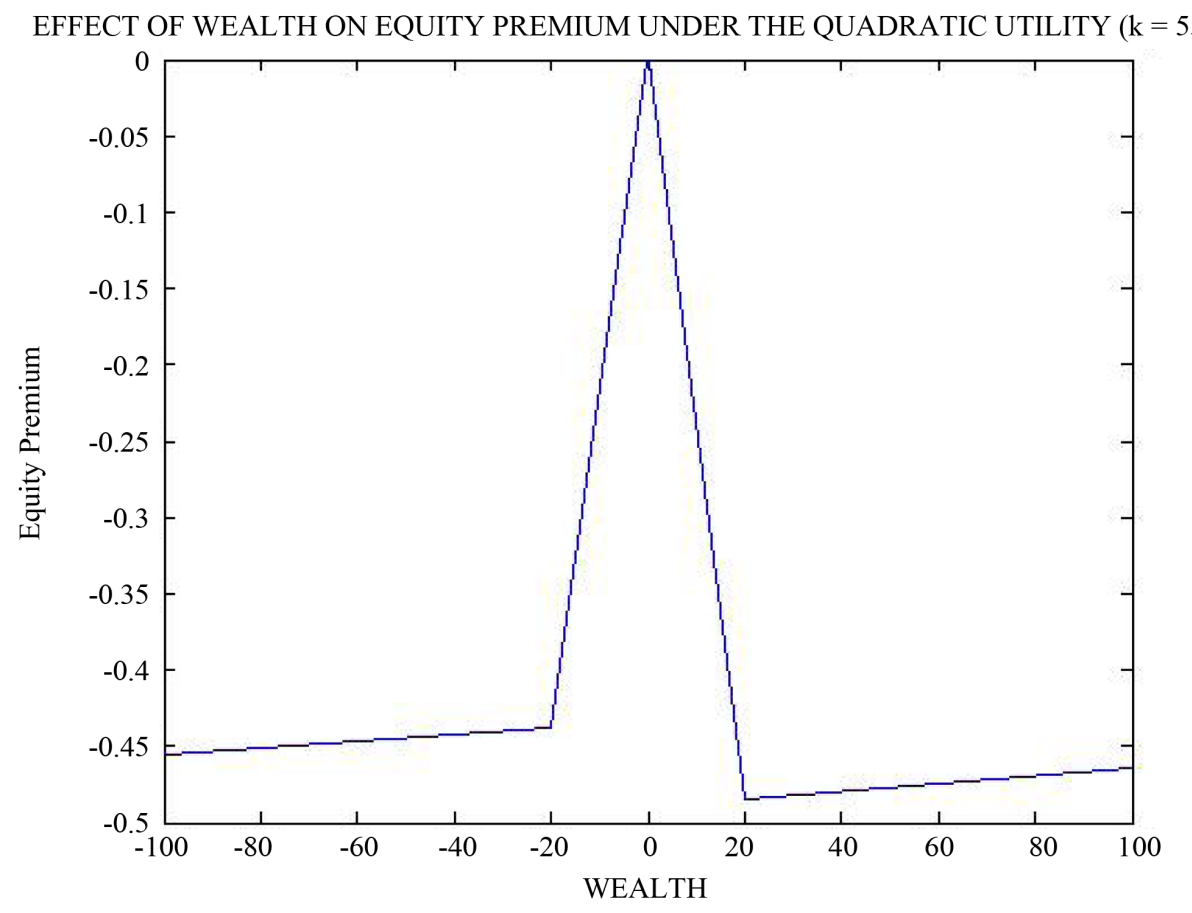

Figure 9. Quadratic utility wealth effect under gamma distribution. 


\section{Acknowledgements}

We thank the Editor and the referee for their comments.

\section{References}

[1] Bates, D.S. (1988) Pricing Options under Jump-Diffusion Processes. The Wharton School, University of Pennsylvania.

[2] Bates, D.S. (1991) The Crash of '87: Was It Expected? The Evidence from Options Markets. Journal of Finance, 46, 1009-1044. http://dx.doi.org/10.1111/j.1540-6261.1991.tb03775.x

[3] Zhang, J.E. and Zhao, H.-M. Asset Pricing under Jump Diffusion. Paper Presented at Quantitative Methods in Finance Conference, 2006, Sydney, Asian Finance Association/Financial Management Association Annual Meeting 2007 in Hong Kong, and 2007 China International Conference in Finance (CICF 2007) in Chengdu, 2006.

[4] Kim, K. and Qian, X. (2007) Convergence of the Binomial Tree Method for Asian Options in Jump-Diffusion Models. Journal of Mathematical Analysis and Applications, 330, 10-23. http://dx.doi.org/10.1016/j.jmaa.2006.07.042

[5] Amin, K.I. (1993) Jump Diffusion Option Valuation in Discrete Time. The Journal of Finance, 48, 1833-1863. http://dx.doi.org/10.1111/j.1540-6261.1993.tb05130.x

[6] Lewis, A.L. (2001) A Simple Option Formula for General Jump-Diffusion and Other Exponential Lévy Processes. Envision Financial Systems and OptionCity.net.

[7] Kou, S.G. (2002) A Jump-Diffusion Model for Option Pricing. Department of Industrial Engineering and Operations Research, Columbia University, New York.

[8] Lyuu, Y.-D. (2002) Financial Engineering and Computation; Principles, Mathematics, Algorithms. Cambridge University Press, Cambridge.

[9] Liu, J., Pan, J. and Wang, T. (2005) An Equilibrium Model of Rare-Event Premium and Its Implication for Option Smirks. Review of Financial Studies, 18, 131-164. http://dx.doi.org/10.1093/rfs/hhi011

[10] Bates, D.S. (2008) The Market for Crash Risk. Journal of Economic Dynamics \& Control, 32, 2291-2321. http://dx.doi.org/10.1016/j.jedc.2007.09.020

[11] Santa-Clara, P. and Yan, S. (2010) Crashes, Volatility, and the Equity Premium: Lessons from S\&P 500 Options. Review of Economics and Statistics, 92, 435-451. http://dx.doi.org/10.1162/rest.2010.11549

[12] Wissem, B., Anand, N.P. and Faouzi, T. (2011) Pricing and Hedging of Asian Option under Jumps. IAENG International Journal of Applied Mathematics, 41, 310-319.

[13] Makate, N. and Sattayatham, P. (2011) Stochastic Volatility Jump-Diffusion Model for Option Pricing. Journal of Mathematical Finance, 1, 90-97. http://dx.doi.org/10.4236/jmf.2011.13012

[14] Zhang, J.E., Zhao, H.M. and Chang, E.C. (2012) Equilibrium Asset and Option Pricing Under Jump Diffusion. Mathematical Finance, 22, 538-568. http://dx.doi.org/10.1111/j.1467-9965.2010.00468.x

[15] Frontczak, R. (2013) Pricing Options in Jump Diffusion Models Using Mellin Transforms. Journal of Mathematical Finance, 3, 366-373. http://dx.doi.org/10.4236/jmf.2013.33037

[16] Sidorov, S.P., Revutskiy, A., Faizliev, A., Korobov, E. and Balash, V. (2014) Stock Volatility Modelling with Augmented GARCH Model with Jumps. IAENG International Journal of Applied Mathematics, 44, 212-220.

[17] Cox, J.C., Ingersoll Jr., J.E. and Ross, S.A. (1985) An Intertemporal General Equilibrium Model of Asset Prices. Econometrica, 53, 363-384. http://dx.doi.org/10.2307/1911241

[18] Bollerslev, T., Gibson, M. and Zhou, H. (2008) Dynamic Estimation of Volatility Risk Premia and Investor Risk Aversion from Option-Implied and Realized Volatilities. Working Paper, Duke University and the Federal Reserve Board.

[19] Bollerslev, T. and Zhou, H. (2007) Expected Stock Returns and Variance Risk Premia. Working Paper, Finance and Economics Discussion Series 2007-11, Board of Governors of the Federal Reserve System (US).

[20] Pan, J. (2002) The Jump-Risk Premia Implicit in Options: Evidence from an Integrated Time-Series Study. Journal of Financial Economics, 63, 3-50. http://dx.doi.org/10.1016/S0304-405X(01)00088-5

[21] Eraker, B. (2004) Do Equity Prices and Volatility Jump? Reconciling Evidence from Sport and Option Prices. The Journal of Finance, 56, 1367-1403. http://dx.doi.org/10.1111/j.1540-6261.2004.00666.x

[22] Broadie, M., Chernov, M. and Johannes, M. (2007) Model Specification and Risk Premiums: Evidence from Future Options. The Journal of Finance, 62, 1453-1490. http://dx.doi.org/10.1111/j.1540-6261.2007.01241.x

[23] Duffie, D., Pan, J. and Singleton, K.J. (2000) Transform Analysis and Asset Pricing for Affine Jump-Diffusions. Econometrica, 68, 1343-1376. http://dx.doi.org/10.1111/1468-0262.00164

[24] Maenhout, P.J. (2004) Robust Portfolio Rules and Asset Pricing. The Review of Financial Studies, 17, $951-983$. 
http://dx.doi.org/10.1093/rfs/hhh003

[25] Mukupa, G.M. and Offen, E.R. (2015) The Impact of Utility Functions on the Equilibrium Equity Premium in a Production Economy with Jump Diffusion. IAENG International Journal of Applied Mathematics, 45, 120-127.

[26] George, M.M. and Offen, E.R. (2016) Equity Premium under Normally Distributed Jump Sizes in a Production Economy with Jumps. International Journal of Applied Mathematics and Statistics, 54, 27-41. 